\title{
Aggregation operators of general aimed information
}

\author{
Doretta Vivona ${ }^{1}$ Maria Divari
}

${ }^{1}$ Dip. di Scienze di Base e Applicate per l'Ingegneria, 16, Via A.Scarpa - 00161 Roma (ITALY)

\begin{abstract}
The aim of this paper is to introduce, by axiomatic way, the measure of the general (i.e. without probability) aimed information for crisp sets.

We traslate the problem in a system of functional equation and we present a class of solutions; in the case of independence we characterize the solution.

Finally, we give some aggregation operators for this form of information measures.
\end{abstract}

Keywords: Information, functional equations, aggregation operators.

\section{Introduction}

In the setting of general (i.e. without probability) information, we shall characterize a measure of information of a crisp variable event, which has the aim to achieve a fixed crisp event. We call this new measure aimed information.

Example: the measure of information about sets of men from different states, who achieve the same diploma.

In the sect. 2 we shall recall some preliminaires following the information theory of Kampé De Feriét and Forte.

The sect. 3 is devoted to the introduction of the aimed information, by axiomatic way, and the statment of the problem in a system of functional equations. For this system we shall find a class of solutions; the solution has been found in the case of independence.

Then, in the sect. 4 some aggregation operators for the aimed information are proposed; finally, in the sect. 5 we give the conclusion.

\section{Preliminaires}

Let $\Omega$ be an abstract space and $\mathcal{A}$ a $\sigma$-algebra of all not-empty subsets of $\Omega, \mathcal{A} \subset \mathcal{P}(\Omega)$, such that $(\Omega, \mathcal{A})$ is measurable. We refer to [7] for all knowledges and operations about crisp sets. We recall the definition of measure of information as in $[8,5]$.

Definition 2.1 The measure $J(\cdot)$ of the general information is a map

$$
J(\cdot): \mathcal{A} \rightarrow[0,+\infty]
$$

such that $\forall A_{1}, A_{2} \in \mathcal{A}$ :
(i) $A_{1} \supset A_{2} \Rightarrow J\left(A_{1}\right) \leq J\left(A_{2}\right)$,
(ii) $J(\emptyset)=+\infty \quad, \quad J(X)=0$,
(iii) $J\left(A_{1} \cap A_{2}\right)=J\left(A_{1}\right)+J\left(A_{2}\right)$, if $A_{1} \cap A_{2} \neq \emptyset$.

As regard to (iii), we assume it as the definition of independence with respect to the information $J$ (shortly $J$-independence).

If $J$ satisfies the previous properties [(i)-(iii)], we call the triple $(\Omega, \mathcal{A}, \mathrm{J})$ information space $[2,5]$.

\section{General aimed information}

Let $S \in \mathcal{A}$ be a fixed set, which represents the object of the events $A \in \mathcal{A}$.

Now, we are ready to give, by axiomatic way, the definition of general aimed information.

Definition 3.1 The general information of $A \in \mathcal{A}$, aimed by a fixed event $S \in \mathcal{A}$ is a map

$$
I(\cdot \rightarrow S): \mathcal{A} \rightarrow[0,+\infty]
$$

such that

$$
\begin{aligned}
& (j) A \supset A^{\prime} \Longrightarrow I(A \rightarrow S) \leq I\left(A^{\prime} \rightarrow S\right), \\
& \forall A, A^{\prime} \in \mathcal{A}, \\
& (j j) I(\emptyset \rightarrow S)=+\infty, \quad I(\Omega \rightarrow S)=0, \\
& (j j j) I((A \cap B) \rightarrow S)=I(A \rightarrow S)+I(B \rightarrow S), \\
& \text { if } A \cap B \neq \emptyset .
\end{aligned}
$$

As regard to (jjj), we assume it as the definition of aimed-independence with respect to the information $I$ (shortly $I$-aimed-independence).

Using these propeties, we shall give a class of general aimed information. Therefore, we plan out a system of functional equations [1], for which we shall present a class of solutions. It is possible to find a solution of the system if the condition of independence has been added.

\section{Statement of the problem}

Let $(\Omega, \mathcal{A}, J)$ be an information space.

Taking into account the previous axiomatic statement, fixed $S \in \mathcal{A}$, we shall looking for a class of aimed measures $I(A \rightarrow S)$, by supposing that $I(A \rightarrow S)$ depends only on $J(A \cap S), J(A), J(S)$. For this reason, fixed $S$, we restrict our definition to the following family:

$$
\mathcal{A}_{\mathrm{S}}=\{\mathrm{A} \in \mathcal{A} / \mathrm{A} \cap \mathrm{S} \neq \emptyset\} ;
$$


at least $\mathcal{A}_{\mathrm{S}}$ contains the whole space $\Omega$, so this family is not empty. We are looking for the aimed information $I(\cdot \rightarrow S)$ as a function:

$$
I(\cdot \rightarrow S): \mathcal{A}_{\mathrm{S}} \rightarrow[0,+\infty]
$$

and a function $\Phi: D \rightarrow[0,+\infty]$, such that

$$
I(A \rightarrow S)=\Phi(J(A \cap S), J(A), J(S)) .
$$

The domain $D$ will be specified later.

Putting $x=J(A \cap S), y=J(A), z=J(S), y^{\prime}=$ $J\left(A^{\prime}\right), t=J(B), x \geq y, x \geq z, x, y, z, x^{\prime}, t \in$ $[0,+\infty]$, with $B \in \mathcal{A}_{\mathrm{S}}$, we recognize that the domain $D$ of the function $\Phi$ is the following

$$
D=\{(x, y, z) / x \geq y, x \geq z\} .
$$

The (1) becomes

$$
I(A \rightarrow S)=\Phi(x, y, z) .
$$

From the properties (j), (jj), (jjj) above, we get the following system of functional equations:

$$
\left\{\begin{array}{l}
\text { (a) } \Phi(x, y, z) \leq \Phi\left(x^{\prime}, y^{\prime}, z\right) \quad x \leq x^{\prime}, y \leq y^{\prime}, \\
\text { (b) } \Phi(+\infty,+\infty, z)=+\infty \\
\text { (c) } \Phi(z, 0, z)=0 \\
\text { (d) } \Phi(y+t+z, y+t, z)= \\
\\
\Phi(y+z, y, z)+\Phi(t+z, t, z) .
\end{array}\right.
$$

For the (d), fixed $S$, and given $A, B \in \mathcal{A}_{\mathrm{S}}$, we suppose that $A, B$ and $S$ are $J$-independent, from (iii) $J(A \cap B \cap S)=J(A)+J(B)+J(S)$. Moreover, by (1), we get

$$
\begin{aligned}
& I((A \cap B) \rightarrow S)=\Phi(J(A \cap B \cap S), J(A \cap B), J(S)) \\
& =\Phi(J(A)+J(B)+J(S), J(A)+J(B), J(S))
\end{aligned}
$$

and

$$
I(B \rightarrow S)=\Phi(J(B \cap S), J(B), J(S)) .
$$

Taking into account (2), we obtain the equation (d) by (1), (3) and (4).

We are looking for an universal solution of the system [(a)-(d)], i.e. a solution of the e quations and the inequality in every proper space.

First, we can give the following result:

Proposition 4.1 A class of solutions of the system $[(\mathrm{a})-(\mathrm{c})]$ is

$$
\Phi_{h}(x, y, z)=h^{-1}[h(x)+h(y)-h(z)],
$$

where $h$ is any continuous, positive, increasing function with $h(0)=0$ and $h(+\infty)=+\infty$.

Proof.The (a) is valid as $h$ is increasing and $x \leq$ $x^{\prime}, y \leq y^{\prime}$.

The (b) and (c) are satisfied from the values of $h$.
By using the $J$ - aimed independence and the form (5) with the hypothesis (on $h$ ) of the Proposition (4.1), the system [(a)-(d)] becomes:

$$
\left\{\begin{array}{l}
\left(a^{\prime}\right) h^{-1}[h(x)+h(y)-h(z)] \leq \\
h^{-1}\left[h\left(x^{\prime}\right)+h\left(y^{\prime}\right)-h(z)\right] \quad x \leq x^{\prime}, y \leq y^{\prime}, \\
\left(b^{\prime}\right) h^{-1}[h(+\infty)+h(+\infty)-h(z)]=+\infty, \\
\left(c^{\prime}\right) h^{-1}[h(z)+h(0)-h(z)]=0, \\
\left(d^{\prime}\right) h^{-1}[h(y+t+z)+h(y+t)-h(z)]= \\
h^{-1}[h(y+z)+h(y)-h(z)]+ \\
h^{-1}[h(t+z)+h(t)-h(z)] .
\end{array}\right.
$$

Proposition 4.2 The solution of the system [(a')$\left.\left(\mathrm{d}^{\prime}\right)\right]$ is

$$
h(x)=c x \quad c>0 .
$$

Proof. It is easy to see that (6) is solution of the system $\left[\left(\mathrm{a}^{\prime}\right)-\left(\mathrm{d}^{\prime}\right)\right]$.

In fact, (a'), (b'), (c') are identically satisfied by (6) through the hyphotesis of $h$. As regards to (d'), putting

$$
\varphi(y, z)=h^{-1}[h(y+z)+h(y)-h(z)],
$$

(d') is

$$
\varphi(y+t, z)=\varphi(y, z)+\varphi(t, z) .
$$

Fized $z=z^{*}$ the (8) is the classical Cauchy equation [1], whose solution is

$$
\varphi\left(y, z^{*}\right)=\lambda\left(z^{*}\right) y
$$

From (7), we get

$$
\begin{aligned}
& \lambda\left(z^{*}\right) y=h^{-1}\left[h\left(y+z^{*}\right)+h(y)-h\left(z^{*}\right)\right], \text { i.e. } \\
& h\left(\lambda\left(z^{*}\right) y\right)=h\left(y+z^{*}\right)+h(y)-h\left(z^{*}\right) .
\end{aligned}
$$

If $z^{*}=0$, as $h(0)=0$, from $(10)$ it is $h(\lambda(0) y)=$ $2 h(y)$, but $h$ is injective, so $\lambda(0)=2$ and it implies $\lambda(z)=2 \forall z$. The function $h$ satisfies the following condition:

$$
h(2 y)=2 h(y) .
$$

From (7), we have obtained 


$$
\begin{gathered}
\varphi(y, z)=2 y=h^{-1}[h(y+z)+h(y)-h(z)], \text { i.e. } \\
h(2 y)=h(y+z)+h(y)-h(z) .
\end{gathered}
$$

The two conditions (11) and (12) imply

$$
h(y+z)=h(y)+h(z) .
$$

We have found again the Cauchy equation, whose solution is $h(x)=c x$, with $c$ real positive number, moreover, the solution of the system $\left[\left(\mathrm{a}^{\prime}\right)-\left(\mathrm{d}^{\prime}\right)\right]$ is (6).

Taking into account the Propositions (4.1) and (4.2), we have the following theorem:

Theorem 4.3 The solution of the system $[(\mathrm{a})-(\mathrm{d})]$ is

$$
\Phi(x, y, z)=x+y-z .
$$

So, we have found a class of $I$-aimed information: from (5) we get

$$
\begin{gathered}
I_{h}(A \rightarrow S)= \\
h^{-1}(h(J(A \cap S))+h(J(A))-h(J(S))),
\end{gathered}
$$

where $h$ is any continuous, positive, increasing function with $h(0)=0$ and $h(+\infty)=+\infty$.

In the case of independence, from (14), we have:

$$
I(A \rightarrow S)=J(A \cap S)+J(A)-J(S) .
$$

\section{Aggregation operators}

We know $[3,4,6,9,10]$ that an aggregation operator is a procedure by which a unique value can be associated to the result obtained through different tests or different data base. The unique value is kind of aritmetic mean. We think that it can be usefully for the applications to give some agggregation operators for aimed general information, too.

From now on, we fix $S \in \mathcal{A}$. Let $\mathcal{I}_{\mathrm{S}} \subset[0,+\infty]^{\mathrm{n}}$ be the family of the aimed general information $I(\cdot \rightarrow$ $S)$.

The aggregation operator $L$ of $n$ aimed general information $I\left(A_{k} \rightarrow S\right), A_{k} \in \mathcal{A}, \mathrm{k}=1, \ldots \mathrm{n}$, is a $\operatorname{map} L: \mathcal{I}_{\mathrm{S}} \longrightarrow[0,+\infty][3,6,10]$ defined by the following properties:

(I) idempotence:

if $I\left(A_{k} \rightarrow S\right)=\lambda, \forall k=1, \ldots n$,

then $L(\underbrace{\lambda, \ldots, \lambda}_{n \text { times }})=\lambda$;

(II) momotonicity:

$I\left(A_{1} \rightarrow S\right) \leq I\left(A_{1}^{\prime} \rightarrow S\right) \Longrightarrow$

$$
\begin{aligned}
& \quad L\left(I\left(A_{1} \rightarrow S\right), \ldots, I\left(A_{k} \rightarrow S\right), \ldots, I\left(A_{n} \rightarrow S\right)\right) \leq \\
& L\left(I\left(A_{1}^{\prime} \rightarrow S\right), \ldots, I\left(A_{k} \rightarrow S\right), \ldots, I\left(A_{n} \rightarrow S\right)\right) ;
\end{aligned}
$$

(III) continuity from below:

$$
\begin{aligned}
& I\left(A_{1 / m} \rightarrow S\right) \nearrow I\left(A_{1} \rightarrow S\right) \Longrightarrow \\
& L\left(I\left(A_{1 / m} \rightarrow S\right), \ldots, I\left(A_{k} \rightarrow S\right), \ldots, I\left(A_{n} \rightarrow S\right)\right) \\
& \nearrow L\left(I\left(A_{1} \rightarrow S\right), \ldots, I\left(A_{k} \rightarrow S\right), \ldots, I\left(A_{n} \rightarrow\right.\right. \\
& S)) .
\end{aligned}
$$

Putting $I\left(A_{k} \rightarrow S\right)=x_{k}, k=1, \ldots, n, I\left(A_{1}^{\prime} \rightarrow\right.$ $S)=x_{1}^{\prime}, I\left(A_{1 / m} \rightarrow S\right)=x_{1 / m}$, we obtain:

$$
\left\{\begin{array}{l}
\left(I^{\prime}\right) L(\underbrace{\lambda, \ldots, \lambda}_{n \text { times }})=\lambda, \\
\left(I I^{\prime}\right) x_{1} \leq x_{1}{ }^{\prime} \Longrightarrow \\
L\left(x_{1}, \ldots, x_{k}, \ldots, x_{n}\right) \leq L\left(x_{1}^{\prime}, \ldots, x_{k}, \ldots, x_{n}\right) \\
\left(I I I^{\prime}\right) x_{1 / m} \nearrow x_{1} \Longrightarrow \\
L\left(x_{1} / m, \ldots, x_{k}, \ldots, x_{n}\right) \nearrow L\left(x_{1}, \ldots, x_{k}, \ldots, x_{n}\right) .
\end{array}\right.
$$

For the solutions of the system [(I')-(III')], we propose the following propositions, whose proofs are immediate:

Proposition 5.1 Two natural solutions of the system [(I')-(III')] are

$$
\begin{aligned}
& L\left(x_{1}, \ldots, x_{k}, \ldots, x_{n}\right)=\bigvee_{k=1}^{n} x_{k} ; \\
& L\left(x_{1}, \ldots, x_{k}, \ldots, x_{n}\right)=\bigwedge_{k=1}^{n} x_{k} .
\end{aligned}
$$

Proposition 5.2 A class of solution of the system [('')-(III') is

$$
L\left(x_{1} \ldots, x_{k}, \ldots, x_{n}\right)=h^{-1}\left(\frac{h\left(x_{1}\right)+\ldots+h\left(x_{n}\right)}{n}\right)
$$

where $h:[0,+\infty] \rightarrow[0,+\infty]$ is any continuous, increasing function $h(0)=0, h(+\infty)=+\infty$.

\section{Remark.}

1) If $h(x)=x$, the aggregation operator $L$ is the aritmetic mean.

2) These results are valid also in fuzzy setting.

\section{Conclusion}

In this paper, we have presented a definition, by axiomatic way, of general aimed information $I(A \rightarrow$ $S)$ in the crisp setting.

If we suppose that this information is linked to $J(A \cap S), J(A), J(S)$, we found a class of solution of a system of functional equations. If we consider the independence, we obtained the solution.

Then, we have given some classes of aggregation operators of these general aimed information. 


\section{Acknowledgment}

This reseach was supported by the research center CRITEVAT of "Sapienza"-University of Rome.

\section{References}

[1] J. ACZÉL: Lectures on Functional Equations and their Applications, (1969) Academic Press, New Jork.

[2] P.Benvenuti: L'opera scientifica, (2004), Roma, Ed.Univ.La Sapienza, Italia.

[3] P.Benvenuti - D.Vivona - M.Divari: Aggregation operators and associated fuzzy mesures, Int.Journ.of Uncertainty, Fuzziness and Knownledge-Based Systems, 2(2001), 197204.

[4] T.Calvo - G.Mayor - R.Mesiar: Aggregation operators, New Trend and Applications, Physisc-Verlag, Heidelberg (2002).

[5] B.FORTE: Measure of information. The general axiomatic theory, R.I.R.O., (1969), 63-90.

[6] M.Grabish, J.-L. Marichal, R.Mesiar, E.PAP: Aggregation Functions, (2009), Cambridge University Press.

[7] P.R.Halmos:Measure Theory, (1965), Van Nostrand Company.

[8] J.KAMpé De FÉRIET, B.ForTE: Information et Probabilité, C.R.Acad.Sc.Paris, 265 (1967), pp. 110-114, 142-146, 350-353.

[9] E.P.KLEMENT: Binary aggregation operators which are bounded by the minimum (a mathematical overview), Proc.AGOP05, (2005), 1316.

[10] D.VIVona - M.DivarI: Aggregation operators for conditional information without probability, Proc.IPMU08, (2008), pp.258-260.

Corresponding author: Doretta Vivona doretta.vivona@uniroma1.it 